\title{
Desarrollo Económico y Seguridad Nacional en el Régimen Militar Argentino
}

\begin{abstract}
Peter G. SN OW es Profesor Titular en la Universidad de Iowa. Fruto de sus continuos viajes por América Latina son sus numerosos libros y folletos. Entre ellos: "Estudios sobre el Comunismo en Bolivia"; "The Political Party System of Chile"; "The Political Party Spectrum in Venezuela"; "Parties and Politics in Argentina: The Elections of 1962 and 1963"; "Argentine Political Parties and the 1966 Revolution"; "Parties and Party Types in Latin American"; "El Radicalismo Argentino"; "El Radicalismo Chileno"*, etc. Actualmente está preparando para el público norteamericano un libro cuyo título es "Interest Group Politics in Argentina".
\end{abstract}

En junio de 1966, las fuerzas armadas argentinas depusieron al Presidente de la Nación elegido constitucionalmente. Era ésta la quinta vez que sucedía lo mismo en los últimos treinta y seis años; sin embargo, pronto se puso de manifiesto el hecho de que éste no iba a ser un golpe militar "típico". Mientras que los gobiernos militares anteriores habían sido de naturaleza esencialmente provisional, el General Juan Carlos Onganía había insistido en que su administración era el agente de la Revolución Argentina, y que él permanecería en el cargo el tiempo necesario para modernizar a la nación. Por primera vez, las fuerzas armadas argentinas asumieron la responsabilidad total frente al desarrollo nacional1 ${ }^{1}$. Ahora, más de seis años después, se han programado elecciones para devolver la autoridad política a representantes elegidos. Sin embargo, parece evidente que los militares no van a renunciar a su responsabilidad en el desarrollo político de la nación. ${ }^{2}$

¿Qué condujo a las fuerzas armadas argentinas a asumir la responsabilidad para con el desarrollo de la nación y aun ahora, evidentemente, a rehusar abandonarla por completo? Esta es una pregunta

* Radicalismo Argentino y Radicalismo Chileno fueron publicados por Editorial Francisco de Aguirre, Buenos Aires-Santiago de Chile, 1972.

${ }^{1}$ Ver La Revolución Argentina. (Buenos Aires: Imprenta del Congreso de la Nación, 1966).

${ }^{2}$ Ver el discurso del Comandante en Jefe del Ejército, Teniente General Alejandro Agustín Lanusse, en Santa Rosa, La Pampa, el 25 de mayo de 1972. 
que no tiene una respuesta única ni simple. Una de las principales razones, sin embargo, parece ser un cambio en la idea militar de seguridad nacional; un concepto que ahora une irrevocablemente la seguridad con el desarrollo económico. Este artículo intenta un breve análisis de este concepto. ${ }^{3}$

A pesar de que la relación entre desarrollo económico y seguridad nacional ha sido popularizada durante los últimos años, no es ciertamente un concepto nuevo. Ya en 1925, el Coronel Luis E. Vicat dijo en un discurso en el Círculo Militar:

"La verdadera defensa nacional es un asunto muy vasto y complejo y puede definirse diciendo que engloba todas aquellas actividades y todas aquellas medidas de previsión necesarias para asegurar la tranquilidad, la prosperidad y la independencia de un país, así como la victoria rápida en caso de conflicto. De nada servirán las instituciones armadas... si en el momento de la acción las armas de fuego llegan a carecer de pólvora y municiones, o no pueden reemplazarse las inutilizadas, o si las tropas no pueden ser transportadas y abastecidas con la rapidez necesaria, o si se llega a carecer de los combustibles, hierro y acero, sin los cuales no pueden moverse barcos, aeroplanos, automóviles, ferrocarriles, ni pueden sostenerse con ventaja las fuerzas combatientes... Debo insistir en la imperiosa necesidad de que modifiquemos todo nuestro actual sistema económico e industrial a fin de que seamos capaces de producir todo lo que necesitaríamos en caso de un conflicto, no solamente para tener la seguridad de no llegar a carecer de nada, sino también para poder considerarnos como verdaderamente independientes de toda tutela extranjera." 4

El Coronel Vicat y otros militares que estaban de acuerdo con él se empeñaban en la industrialización como un medio para aumentar la seguridad nacional, pero estaban evidentemente a favor de que esta industrialización fuera planeada y ejecutada por civiles.

Sin embargo, en 1941, los militares pidieron -y obtuvieron-, una gran parte de responsabilidad en este dominio. La ley 12.709 del 26 de setiembre de 1941 creó la Dirección General de Fabricaciones Militares, cuyas funciones eran "realizar investigaciones con el fin de evaluar las posibilidades industriales del país; elaborar materiales y elementos de guerra; realizar explotaciones mineras; construir

sEs necesario admitir que lo que yo creo ser un plan para la acción militar sea en cambio una racionalización.

* Citado en Rodolfo Puiggrós, Las izquierdas y el problema nacional. (Buenos Aires: Jorge Alvarez, editor, 1967), pp. 28-29. 
las obras de infraestructura necesarias; fomentar industrias afines."

Bajo el liderazgo de su primer director, el Coronel Manuel $\mathrm{N}$. Savio, Fabricaciones Militares pronto jugó un papel dominante en la producción nacional de acero. Aun hoy, posee la mayoría de las acciones de la Sociedad Mixta Siderurgia Argentina (SOMISA), que, a su vez, tiene casi el monopolio sobre la producción de productos de acero. ${ }^{6}$

Durante la última década, algunos líderes militares han dejado bien claro su concepto de la relación que existe (incluso necesariamente) entre el desarrollo económico y la seguridad nacional. El corolario de este concepto es, por supuesto, la responsabilidad de los militares por ambos aspectos. Los más conspicuos exponentes de esta noción son los Generales de División en retiro Juan Enrique Guglialmelli, Osiris Guillermo Villegas y Juan N. E. Tavícoli y el Comodoro en retiro Juan José Güiraldes ${ }^{7}$. Ya en diciembre de 1964 el General Guglialmelli dijo en un discurso en el Centro de Altos Estudios Militares:

"El desarrollo económico-social no sólo es un imperativo de la solidaridad para crear trabajo y bienestar; es una necesidad impostergable de la seguridad nacional. ${ }^{\mathrm{s}}$

Cuatro años más tarde el General Villegas escribió: "No puede haber seguridad sin desarrollo, como tampoco desarrollo sin seguridad."8 Aproximadamente al mismo tiempo, el General Iavícoli escribió: "El desarrollo es a la seguridad lo que la causa al efecto, el origen a la consecuencia, lo principal a lo secundario; sin desarrollo, la seguridad es utopía."10

'Norberto Ceresole, Ejército y Politica Nacionalista. (Buenos Aires: Editorial Sudestada, 1968), p. 101. El equivalente de Fabricaciones Militares en la Armada y la Fuerza Aérea son Astilleros y Fábricas Navales del Estado (AFNE) y Dirección Nacional de Fabricaciones e Investigaciones Aeronáuticas (DINFIA).

oToda la propaganda actual de Fabricaciones Militares dice que: "investiga las posibilidades industriales del país, alienta e impulsa el desarrollo nacional, $y$ contribuy'e a la seguridad nacional."

"Posiblemente no es una coincidencia el hecho de que cuatro de estos hombres asistieron al Colegio Militar a fines de los años treinta. Deben haber tenido los mismos instructores, y probablemente, haber entablado amistad en los comienzos de la carrera militar.

${ }^{8}$ Este discurso se publicó en la Revista de la Escuela Superior de Guerra. Nr. 356 (1964), p. 113 .

"General de División Osiris Guillermo Villegas, "Seguridad, Política, Estrategia." Temas Militares I: 4 (Febrero 1968), p. 5.

${ }^{10}$ General de División Juan N. E. Iavícoli, "La interrelación del Desarrollo y la Seguridad en el Estado Moderno", Temas Militares I: 2 (Enero-Febrero 1967), p. 82. 
El Comodoro Güiraldes cambió la terminología sólo ligeramente cuando escribió: "La idea de desarrollo y la de país desarrollado no pueden desprenderse de la idea de sociedad industrial." 11

Estos desarrollistas tienden a igualar el desarrollo con la-industrialización total. Están convencidos de que una economía nacional tal como la de Argentina, que está basada en exportaciones agrícolas, "no resuelve a mediano ni a largo plazo los problemas fundamentales de la población, y crea vulnerabilidades sustanciales para la seguridad nacional... Un país que no tenga las industrias básicas es un país castrado, porque las industrias básicas significan a los pueblos lo que los elementos viriles al vacuno. Nosotros no podemos pretender ni resolver los problemas de la economía a largo plazo, ni los problemas de la seguridad, ni los problemas de nuestro valor relativo en el ámbito natural que nos es propio en el cono sur de Latinoamérica, si no tenemos ese tipo de país."12

La seguridad nacional es definida por los desarrollistas en los términos de la Ley de Defensa Nacional de 1967, que dice: "La seguridad nacional es la situación en la cual los intereses vitales de la $\mathrm{Na}$. ción se hallan a cubierto de interferencias y perturbaciones sustanciales."13

De acuerdo con el General Villegas, esos intereses vitales de la nación incluyen: "El promover la explotación de sus recursos naturales, impulsar el desarrollo de las industrias básicas, dotar al país de una infraestructura eficiente."14 pública, el desarrollo se convierte en la hora actual, en condición indispensable para la seguridad; porque el desarrollo proporciona los factores con que se actúa en la protección de Ios intereses vitales de la Nación. La armonía social, de la que depende el orden interno, está condicionada, entre otros elementos, a una justa distribución de la riqueza, sólo posible cuando existen las condiciones ambientales necesarias para crear dicha riqueza, lo cual es más fácil, si el país tiene desenvueltas sus aptitudes y capacidad para lograr sus decisiones fundamentales, contener los embates de otros países, cuando no de grupos privados internacionales - de intereses o de

${ }^{11 C o m o d o r o ~ J u a n ~ J o s e ́ ~ G u ̈ i r a l d e s, ~ " F u e r z a s ~ A r m a d a s ~ y ~ D e s a r r o l l o ", ~ T e m a s ~}$ Militares, I: (Sept.-Octubre 1966), p. 52.

${ }^{12}$ General de División Juan E. Guglialmelli, 120 Dias en el Gobierno. (Buenos Aires: Edición del Autor, 1971), pp. 42-43. Los 120 dias a que se hace referencia son aquellos en que el autor fue Secretario General de CONADE, entre el 30 de junio y el 3 de noviembre, 1970 .

${ }^{13}$ Ley 16.970, artículo 2.

${ }^{14}$ General de División Osiris Guillermo Villegas "Seguridad, Politica, Estrategia", Temas Militares. I: 4 (Febrero 1968), p. 4. 
ideologías- que tienen posibilidades de poner a su servicio importantes factores internos y/o externos de poder." 15

Los desarrollistas no se contentan con destacar la relación entre desarrollo y seguridad, sino que continúan insistiendo en que las fuerzas armadas deberían asumir un rol importante, si no dominante, en el proceso de desarrollo. Implícita en el material citado hasta ahora está la idea de que, puesto que el desarrollo es un prerrequisito para la seguridad $y$, puesto que la preservación de la seguridad es una función militar, los militares deben también asumir responsabilidad frente al desarrollo. Algunos van más allá, afirmando que varias de las características del poder militar hacen singularmente adecuada la vigilancia del proceso de desarrollo.

Por ejemplo, el Comodoro Güiraldes dice:

"Si. debe destacarse la función de las fuerzas armadas con características propias, es porque en países como el nuestro, donde la racionalización, las disciplina, la jerarquización y la organización son notas que no caracterizan a los grupos públicos o privados que atienden los distintos servicios que la sociedad reclama, las Fuerzas Armadas, que por esencia responden a esas características, tienen un papel ejemplar a cumplir. ${ }^{26}$

El General Guglialmelli dice esencialmente la misma cosa, a pesar de que lleva esta idea un paso más adelante:

"Las Fuerzas Armadas constituyen un núcleo particularmente cohesionado, representativo de toda la comunidad, capaz de individualizar y enfrentar, dentro y fuera de las fronteras, al enemigo nacional." 17

Una vez que el subdesarrollo ha sido definido como un "enemigo nacional", la responsabilidad de los militares por el proceso de desarrollo no sólo se vuelve legítima, sino un deber. A este respecto, el General Guglialmelli es bastante explícito:

"El enemigo de la Nación en ciernes es nuestra falta de desarrollo; sobre esta circunstancia elaboran su estrategia los monopolios. Corresponde a las Fuerzas Armadas, como siempre que se trata de ábatir a un enemigo, un papel de vanguardia. Su lucha, que es la lucha de la Nación, tiene que librarse construyendo las bases materiales y espirituales del desarrollo." 18

${ }^{15}$ General de División Osiris Guillermo Villegas, Politicas y Estrategias para el Desarrollo y la Seguridad Nacional. (Buenos Aires: Editorial Pleamar, 1969), p. 109.

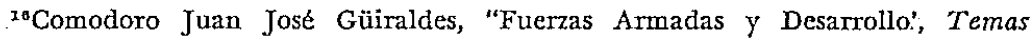
Militares. X: 1 (Sept.-Octubre 1966), p. 55.

- ${ }^{27}$ General de División Juan E. Guglialmelli, "Responsabilidad de las Fuerzas Armadas en la Revolución Nacional", Estrategia. Nr. 4 (Nov.-Dic. 1969), p. 8.

${ }^{18}$ General de División Juan E. Guglialmelli, 120 Dias en el Gobierno. (Buenos Aires: Edición del Autor, 197.1), p. 83. 
En otro lugar, el General Guglialmelli dice que el desarrollo es un medio para prevenir la violencia - otra vez una responsabilidad militar legítima:

"El problema para las Fuerzas Armadas no puede ni- debe limitarse a proporcionar la ley y el orden, ni circunscribirse al estudio y la planificación de las medidas represivas para el caso que la violencia estalle. Más importante que lo antedicho es comprender las necesidades exigidas por el desarrollo, e impulsarlo para evitar que esa violencia se produzca."19

No son sólo los desarrollistas dentro del ejército quienes insisten acerca de la responsabilidad militar por el desarrollo económico. Esta posición es compartida también por muchos oficiales nacionalistas: Mientras los nacionalistas están de acuerdo en que el desarrollo es esencial para la seguridad nacional, insisten en que ninguno de ellos pueden ser posibles dentro del marco de la democracia liberal. Uno de los voceros más articulados de los nacionalistas, el General Eduardo Juan Uriburu, ha escrito recientemente:

"La experiencia que hemos ganado durante largos períodos de democracia anglosajona y de un estado liberal es que las consideraciones básicas para el desarrollo y la seguridad nacional son pospuestas para solucionar las necesidades electorales inmediatas, dilatando de este modo la marcha acelerada del país, que demandan los tiempos en que vivimos. La solución se encontrará en un estado nacionalsocialista que, basado en un planeamiento metódico, impulse el desarrollo, sin tomar en cuenta los costos, porque los resultados repagarán con intereses los sacrificios demandados durante la ejecución de esos planes." 20

Aun aquellos oficiales que por largo tiempo han sido partidarios de un cuerpo militar totalmente profesional y apolítico están prontos a admitir que las fuerzas armadas tienen que jugar un papel legítimo en el campo del desarrollo. Por ejemplo, el General Benjamín

${ }^{20}$ General de División Juan E. Guglialmelli, "Seguridad y Desarrollo", Temas Militares. I: 2 (enero-febrero), p. II.

${ }^{20}$ General de División Eduardo Juan Uriburu, "El equipamiento de las Fuerzas Armadas y su relación directa con el desarrollo nacional", Estrategia. Nr. 13-14 (nov.-dic. 1971 a enero-febr. 1972), pp. 105-106. Ver también su obra El Plan Europa. Un intento de liberación nacional. (Buenos Aires: Cruz y Fierro.Editores, 1970). Una posición nacionalista aún más extrema es la planteada por el ex Teniente Corónel Florentino .Dlaz Loza en Las armas de la revolución. (Buenos Aires: A..Peña Lillo, 1972) y. su "Argentina, conyuntura histórica del siglo xx", Estrategia, Nrs. 13-14 (nov.-dic. 1971 a enero-febr. 1972), pp. 109-116. 
Peter G. Snow / Desarrollo económico y seguridad nacional

Rattenbach, probablemente el más conocido oponente de la intervención militar en política dice:

"Se puede decir que las Fuerzas Armadas tienen por misión hoy día no sólo la defensa exterior y la seguridad interior, sino también la contribución al desarrollo de la nación, tanto en el aspecto económico y social como en el cultural y político."21

Y aun en un artículo cuyo propósito es explicar por qué las fuerzas armadas deben permanecer fuera del dominio de la política, el General Rattenbach dice:

"La intervención de las Fuerzas Armadas, en épocas normales, en las esferas de la industria, de las comunicaciones y la educación se realiza en nuestro país ya desde hace tiempo. Nunca fue juzgada como una intromisión, sino como una contribución lógica al desarrollo y siempre fue bien vista por el resto de la sociedad."22.

A pesar de que todos los líderes militares citados anteriormente están ahora en retiro, no hay razón para creer que sus ideas difieren sustancialmente de aquellas sostenidas por los oficiales que están ahora en servicio activo. Hay por lo menos dos factores que conducen a creer que el nuevo concepto de seguridad nacional está todavía muy en boga, y que continuará estándolo. Primeramente, la relación entre desarrollo y seguridad ha sido institucionalizada hasta un cierto punto con la creación en 1966 del Consejo Nacional de Des̀arrollo (CONADE) y del Consejo Nacional de Seguridad (CONASE). Mientras las actividades diarias de estos consejos están bajo la supervisión de Secretarios Generales con rango ministerial, estos están presididos por el Presidente de la Nación, e incluye en todas las deliberaciones a todos los ministros y a los Comandantes en Jefe de las tres fuerzas armadas. En adición a sus deberes obvios, CONADE y CONASE también llevan a cabo funciones que son esencialmente legislativas. Antes de su promulgación, todas las leyes son enviadas a CONADE para que se determinen sus efectos sobre el desarrollo èconómico y a CONASE para un examen de sus efectos sobre la seguridad nacional. Cada consejo tiene el poder de veto suspensivo, puesto que las leyes consideradas en detrimento del desarrollo $y / 0$ de la seguridad son devueltas a los ministros responsables para su revisión.

De mayor importancia aún es, quizá, la doctrina de seguridad

${ }^{21}$ Teniente General Benjamín Rattenbach, "El Profesionalismo Mrilitar en el Ejército Argentino", Temas Militares. I: 3 (marzo-abril 1967), p: 15.

${ }^{22}$ Teniente General Benjamin Rattenbach, "Las Fuerzas Armadas y la política", Primera Plana. Nr. 243 (22 de agosto de 1967), p. 36. 
nacional enseñada en la Escuela Nacional de Guerra. De acuerdo con uno de los textos que se usan allí, la seguridad es concebida: "No como un medio para mantener incólume la soberanía territorial detrás de fronteras rígidamente amojonadas, sino como una situación, graduable por cierto, en la cual los intereses vitales de la Nación se hallan a cubierto de interferencias y perturbaciones -internas o externas, violentas o no violentas, abiertas o subrepticias- que pueden neutralizar o postrar el desarrollo, y por ende la existencia misma de la Nación o su soberanía... El moderno concepto de seguridad es por lo tanto mucho más amplio que el de Defensa Nacional, que ès el "conjunto de medidas que el Estado adopta para lograr la Seguridad Nacional". Dicho con otras palabras; mientras la Defensa Nacional comprende las medidas, la seguridad es la situación resultante de la aplicación de todas las medidas que tienden a preservar y asegurar el acervo espiritual y los bienes materiales de la Nación..... Esa dosis de seguridad debe ser la situación resultante de la aplicación de muy acertadas medidas en el campo de lo social, de lo económico, o de lo educacional, que mediante dinámicas realizaciones de signo positivo en todos los estratos sociales nacionales, nieguen las pautas explotadas por el enemigo $y$ que a la postre arrojen como saldo, un adecuado grado de confianza y de tranquilidad en lo social, en lo económico, en lo educacional, o en todas juntas. Es decir, una situación de seguridad que interesa a toda la Nación y que permite continuar su ininterrumpido desarrollo... Tal es el modemo concepto de seguridad que reclama, por sobre todo, su cabal comprehensión y una armonización en el desarrollo que es su factor fundamental, al que sirve y del cual se sirve. Por ello, la Seguridad Nacional, en su sentido más profundo, no es un concepto puramente militar, tiene un alcance mucho más amplio aún; es parte de la Política Nacional, puesto que se vincula con todos los aspectos de la vida de nuestra sociedad y por ende, de los intereses vitales de la Nación Argentina." 23

El solo hecho de que esto se enseñe en la Escuela Nacional de Guerra es, probablemente, suficiente evidencia para predecir que el concepto desarrollo-seguridad dominará aún por algún tiempo el pensamiento militar, y como resultado de ello, que los militares continuarán exigiendo un mayor papel en el desarrollo económico de la nación.

${ }^{23}$ "La Seguridad Nacional: Un Concepto de Palpitante Actualidad", Estrategia. Nr. 4 (nov.dic. 1969), pp. 133-134. De acuerdo con el editor de Estrategia, este texto fue proporcionado por el General Joaquín A. Aguilar Pinedo, entonces Director de la Escuela Nacional de Guerra. 